\title{
In vivo isolation of circulating tumor cells in patients with different stages of prostate cancer
}

\author{
GERIT THEIL $^{1}$, CATRIN BOEHM ${ }^{1}$, KERSTEN FISCHER $^{1}$, JOANNA BIALEK $^{1}$, RASCHID HODA $^{1}$, \\ EKKEHARD WEBER $^{2}$, SANDRA SCHÖNBURG ${ }^{1}$, FELIX KAWAN ${ }^{1}$ and PAOLO FORNARA ${ }^{1}$ \\ ${ }^{1}$ Medical Faculty of Martin Luther University Halle-Wittenberg, University Clinic and \\ Outpatient Clinic for Urology, D-06120 Halle (Saale); ${ }^{2}$ Institute of Physiological Chemistry, \\ Medical Faculty of Martin Luther University Halle-Wittenberg, D-06120 Halle (Saale), Germany
}

Received November 26, 2020; Accepted February 10, 2021

DOI: $10.3892 / 01.2021 .12618$

\begin{abstract}
Circulating tumor cells (CTCs) provide accurate information on the clinical stage of cancer progression. The present study examined the clinical validity and feasibility of a new medical device for the in vivo isolation of CTCs from the blood of patients with prostate cancer (PCa). The GILUPI CellCollector ${ }^{\circledR}$ (DC01) was applied in 188 cases. The CTC/prostate-specific antigen (PSA) profile of each patient was checked for therapeutic monitoring of patients with PCa. The CellCollector, which is a unique in vivo approach for the isolation of CTCs, was compared with the CellSearch ${ }^{\circledR}$ system, which is the current standard. Overall survival (OS) and diagnostic performance were evaluated. By in vivo isolation, 78.9\% (56/71) of patients with metastatic disease (PCa-m) and 46.3\% (24/53) of patients with localized disease (PCa-l) had $\geq 1$ captured CTC. Kaplan-Meier analysis revealed that patients with PCa-m that had $\geq 5$ CTCs had a significantly different OS compared with those with $<5$ CTCs (27.5 months vs. 37 months; HR 2.6; 95\% CI 0.78-8.3). Patients with a higher number of CTCs at all time-points had the shortest median OS of 25 months (HR 1.9; 95\% CI 0.4-11.6). The effectiveness of CTC isolation technologies demonstrated that in $65.7 \%$ of the applications, patients with cancer were positive for CTCs using the CellCollector. By contrast, the CellSearch system detected CTCs in $44.4 \%$ of applications. In vivo isolation of CTCs demonstrated the clinical viability of the CellCollector, related to the current standard for the isolation of CTCs from patients with $\mathrm{PCa}$. The advantage of the in vivo device is that it overcomes the blood volume limitations of other CTC assays. Furthermore, the present study revealed that the CellCollector was well tolerated, and no adverse events (AEs) or serious AEs were reported.
\end{abstract}

Correspondence to: Dr Gerit Theil, Medical Faculty of Martin Luther University Halle-Wittenberg, University Clinic and Outpatient Clinic for Urology, Ernst-Grube-Str. 40, D-06120 Halle (Saale), Germany

E-mail: gerit.theil@uk-halle.de

Key words: CellCollector ${ }^{\circledR}$, circulating tumor cells, in vivo isolation, prostate cancer, liquid biopsy

\section{Introduction}

Prostate cancer (PCa) is the fifth leading cause of death in men, with 359,500 deaths worldwide in 2018 (1). The disease phenotypes varied from indolent to aggressive. The local stage is potentially curable with local therapy and shows a 5 -year survival rate of nearly $100 \%$, compared with $29.8 \%$ for metastatic cases (1). One challenge for clinicians is to determine the optimal sequencing therapies for patients who present intermediate, high-risk localized, locally advanced or metastatic prostate cancer ( $\mathrm{mPCa}$ ) to minimize overtreatment and improve outcomes. Thus, early and precise detection of cancer is important for decreasing patient mortality. In addition, the current therapeutic landscape offers the patient an individualized treatment approach. Nevertheless, the treatment of mPCa is becoming increasingly complex (2). The risk of overdiagnosis and overtreatment remains and has a negative impact on the quality of life of men with $\mathrm{PCa}$ (3). One of the greatest challenges in the current management of $\mathrm{PCa}$ is adequate assessment of the response to treatment. Prostate-specific antigen (PSA) as a tumor marker for prostate cancer has limitations as a surrogate for survival end points because of insufficient sensitivity and specificity (4). Additionally, PSA determination is not an adequate marker for the evaluation of treatment response. However, personalizing PCa treatment with a biomarker, such as circulating tumor cells (CTCs), offers the possibility to create risk-adapted strategies to optimize patient care. CTCs represent a minimally invasive source of spreading tumor cells and provide important clinical information for the individual patient's treatment in terms of monitoring metastasis, evaluating the efficacy of treatment, and/or facilitating the early detection of treatment resistance (5-7). In recent reports, detection of androgen-receptor splice variant 7 (AR-V7) in pooled CTCs of men with progressive metastatic castration-resistant prostate cancer (mCRPC) was associated with resistance to the androgen receptor inhibitors abiraterone and enzalutamide. This finding shows that CTCs can provide insights into drivers of tumor growth in patients and into the pharmacodynamics effects of targeted therapies $(4,8)$.

However, it remains difficult to isolate and characterize CTCs because of their rarity (1-10 CTCs per ml blood) and 
heterogeneous phenotype (9). Additionally, CTCs are present in a large background of hematopoietic cells. In 2004, the CellSearch ${ }^{\circledR}$ system was the first US Food and Drug Administration (FDA)-authorized system for the enumeration of CTCs in $7.5 \mathrm{ml}$ of blood. Clinical studies demonstrated that CTCs captured with the CellSearch system were clearly associated with poor patient outcomes $(10,11)$. At present, several platforms have been developed to detect CTCs. CTC detection can be achieved based on physical and biological properties $(12,13)$.

In a previous study, we evaluated the CellCollector ${ }^{\circledR}$ (GILUPI CellCollector), an in vivo approach initially ex vivo (in vivo was not allowed at this time) in blood samples from PCa patients. Our results showed that the CellCollector could be applied for the sensitive isolation and molecular characterization of CTCs ex vivo (14). To date, other study has evaluated the CellCollector in single-center trails in patients with breast, lung, high-risk PCa and neuroendocrine tumors in small cohorts (15-17).

In the present study, we validated the CellCollector, which allowed in vivo isolation of CTCs directly from the cubital vein in a cohort of prostate cancer patients in different clinical stages and control groups. This included monitoring prostate cancer patients during treatment for a clinical response correlated to CTC counts and comparison of CellCollector results to those from the CellSearch System.

\section{Materials and methods}

Study population and clinical information. The patients provided written informed consent and were enrolled at University Clinic and Outpatient Clinic for Urology, Medical Faculty of Martin Luther University Halle-Wittenberg from February 2011 to March 2012. The medical faculty ethics committee of Martin Luther University Halle-Wittenberg approved the study protocol. Furthermore, we obtained a permit from the Federal Institute for Drug and Medical Devices (Germany, BfArM).

The study population consisted of 14 metastasized (PCa-m) and 21 localized (PCa-1) PCa patients. A control group of 16 men with benign prostate hypertrophy $(\mathrm{BPH})$ and a second control group of 20 women were also included (Table I). The patients were required to have histologically proven prostate cancer in the localized group and documented metastases, as confirmed by computed tomography (CT), in the metastasized group. All patients had PSA levels determined at every time point of CTC isolation.

In vivo CTC isolation. The CellCollector consists of a $160-\mathrm{mm}$ sterile steel wire with a $20-\mathrm{mm}$ functionalized tip containing epithelial-cell adhesion molecule (EpCAM) antibodies on its surface. The antibodies are covalently bonded to a hydrogel that is linked to a gold layer (Fig. 1). The wire was inserted into the cubital vein through a $20 \mathrm{G}$ cannula and remained in place for $30 \mathrm{~min}$. Then, the CellCollector was washed three times with phosphate-buffered saline (PBS), and captured cells were fixed with $100 \%$ acetone for $10 \mathrm{~min}$ at room temperature and blocked with $3 \%$ bovine serum albumin/PBS for $30 \mathrm{~min}$. The captured cells were identified by immunofluorescence staining, and the CellCollector was examined for fixed cells using a Nikon microscope (TE2000-E) at 20x magnification. The images were digitally processed with ImageJ software by altering the contrast and brightness in accordance with Nature Publishing Guidelines.

CellSearch system. Blood samples were collected into $7.5 \mathrm{ml}$ CellSave tubes. These samples remained stable for $96 \mathrm{~h}$ at room temperature and were sent overnight to the University Medical Center Hamburg-Eppendorf. CTCs were isolated using EpCAM-functionalized immunomagnetic beads with a semiautomated workflow that included enrichment, fluorescent labeling/characterization and automated fluorescence imaging of the rare cell population $(9,18,19)$.

CTC enumeration and morphology. CTC enumeration and identification for both isolation technologies were based on identical criteria. Isolated cells and/or clusters of immunostained cells of interest were examined by a blinded experienced researcher. EpCAM-positive cells were defined as CTCs with the following cytology-based FDA definition: i) size $\geq 4 \mu \mathrm{m}$, ii) visible cytoplasm, iii) high nuclear/cytoplasm ratio, iv) positive fluorescent staining of CK 8, 18, and 19 with negative staining of CD45, and v) $50 \%$ of the nucleus contained within the CK border (20).

Statistical evaluation. Since limited data regarding the CellCollector were available at the time of the study design, no formal sample size calculations were performed. Therefore, our analyses were exploratory in nature. We compared the CTC counts between the control group and the PCa-l and PCa-m groups using Kruskal-Wallis test followed by Dunn's multiple comparison test. The overall survival rates were calculated using CTCs value at baseline and follow up visits. The log-rank test was used for comparing the Kaplan-Meier survival curves. For all analyses, $\mathrm{P}<0.05$ was considered statistically significant. The accuracy of the CTC counts and PSA level were evaluated by receiver operating characteristic (ROC) analysis. Analyses were performed using GraphPad Prism version 6.

\section{Results}

Study population and clinical information. The baseline characteristics and clinical parameters of the different study groups are summarized in Table I. In total, 71 study subjects were enrolled in our trial, and $92.8 \%$ of the 14 PCa-m patients received chemotherapy. Ten prostate cancer patients $(71.4 \%)$ were treated with androgen deprivation therapy (ADT). The primary therapy for all $21 \mathrm{PCa}-1$ patients was radical prostatectomy (RP). Five patients (18.5\%) received postoperative radiation. All patients with benign prostatic hyperplasia in our control group were treated with transurethral resection of the prostate. The second control group consisted of healthy women. A schedule of the in vivo application and the comparison method is presented in Fig. 2.

In vivo CTC isolation. Overall, 188 CellCollector applications were included in the analysis (Fig. 3). The CellCollector was well tolerated, and no adverse events (AEs) or serious adverse events (SAEs) were reported. In the metastatic group, 78.9\% $(n=57)$ of the 71 applications were positive for $\geq 1$ CTCs. Among 
Table I. Study population demographics.

\begin{tabular}{|c|c|c|c|c|}
\hline \multirow[b]{2}{*}{ Characteristics } & \multicolumn{2}{|c|}{ Prostate cancer } & \multicolumn{2}{|c|}{ Control group } \\
\hline & Metastatic & Localized & BPH & Women \\
\hline Patients, $\mathrm{n}$ & 14 & 21 & 16 & 20 \\
\hline Median age (range), years & $52(53-79)$ & $59(56-72)$ & $67(58-83)$ & $25.5(19-38)$ \\
\hline Ethnicity & Caucasian & Caucasian & Caucasian & Caucasian \\
\hline \multicolumn{5}{|l|}{ Gleason score at diagnosis } \\
\hline$\leq 7, \mathrm{n}(\%)$ & $2(14.30)$ & $18(85.7)$ & & \\
\hline$>7, \mathrm{n}(\%)$ & $12(85.70)$ & $3(14.3)$ & & \\
\hline $\begin{array}{l}\text { Median PSA at baseline, } \\
\mathrm{ng} / \mathrm{ml} \text { (range) }\end{array}$ & $23.6(3.5-1120)$ & $7.91(1.8-39.5)$ & $1.9(0.41-15.9)$ & \\
\hline Median PSA, ng/ml (range) & $21.7(0.04-1120)$ & $0.04(0.04-3.3)$ & $0.4(0.04-3.1)$ & \\
\hline \multicolumn{5}{|l|}{ Primary therapy } \\
\hline TURP, n (\%) & & & $16(100)$ & \\
\hline Surgery (RP), n (\%) & $2(13.3)$ & $21(100)$ & & \\
\hline Radiation, n (\%) & $9(60.0)$ & $5(18.5)$ & & \\
\hline \multicolumn{5}{|l|}{ Systemic therapy } \\
\hline Androgen treatment, $\mathrm{n}(\%)$ & $10(71.4)$ & $2(7.1)$ & & \\
\hline Chemotherapy, n (\%) & $13(92.8)$ & & & \\
\hline \multicolumn{5}{|l|}{ Site of metastatic disease } \\
\hline Bone, n (\%) & $12(86.7)$ & & & \\
\hline Lymph node, n (\%) & $6(42.9)$ & & & \\
\hline Other soft tissue, $\mathrm{n}(\%)$ & $3(21.4)$ & & & \\
\hline
\end{tabular}

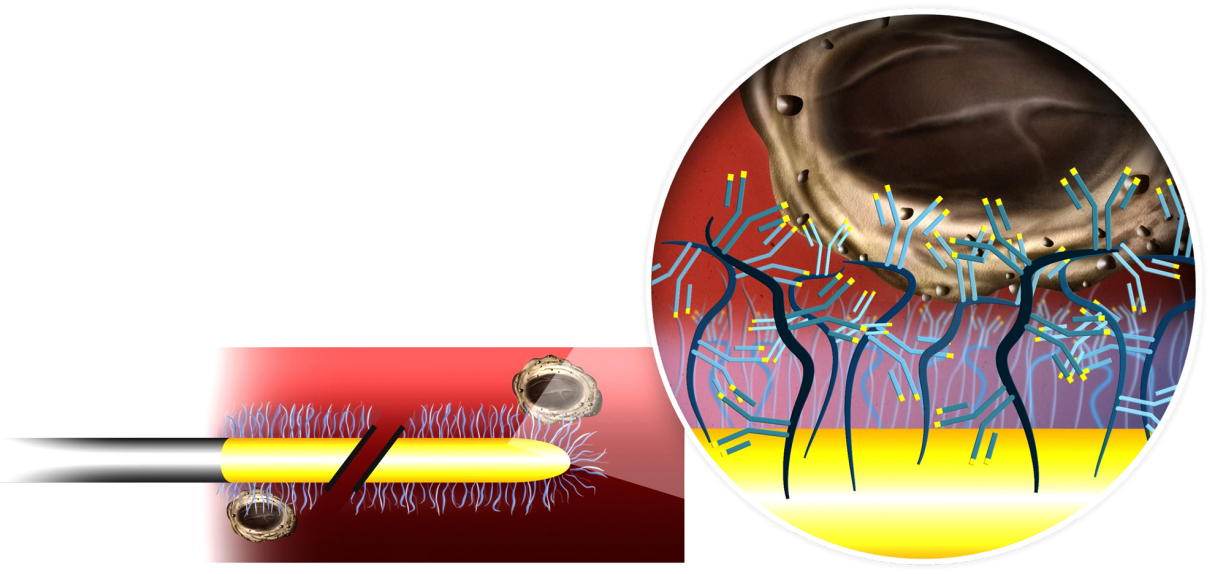

Functionalized surface

Tumor cell

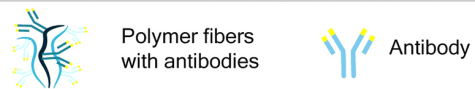

Figure 1. Schematic of the CellCollector ${ }^{\circledast}$. CTC capture process is achieved by using an antibody against EpCAM protein. CTC, circulating tumor cells; EpCAM, epithelial-cell adhesion molecule.

the metastatic patients with detectable CTCs, the median CTC count was 4 (range, $0-820$ ), and the mean CTC count was 27. In the localized group, $45.3 \%(n=24)$ of the 53 CellCollector applications were positive for CTCs. Most of the identified CTCs were single cells, and cell clusters were rarely present. Among the CTC-positive localized PCa patients, the CTC median count was 0 (range, 0-9.0) and the mean CTC count was 1.45, and the CTC count was significantly different $(\mathrm{P}<0.0001)$ between the cancer groups (Fig. 3). A total of $70.7 \%(n=29)$ of the BPH patients and $85 \%(n=17)$ of the women in our control group were negative for CTCs. The median CTC count of the BPH patients was 0 (range, 0-13); a median CTC value of 0 (range, 0-3) was 


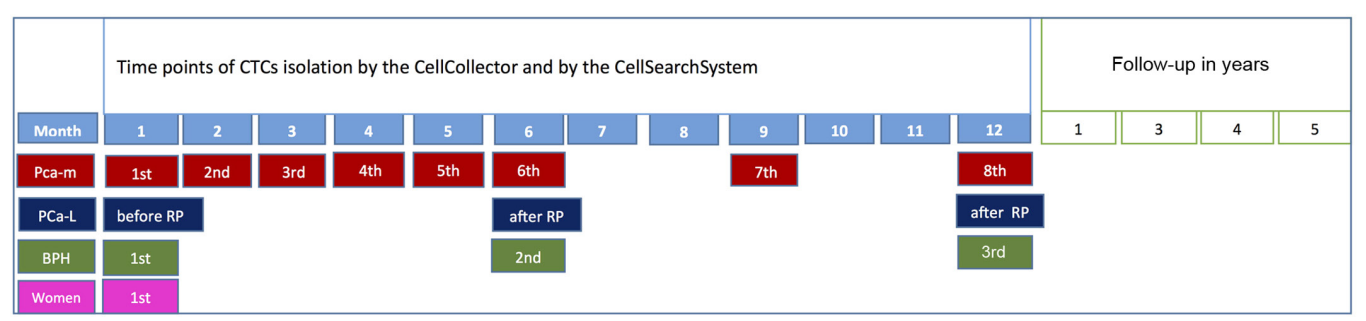

Figure 2. Study design. RP, radical prostatectomy; BPH, benign prostatic hyperplasia; PCa-m, prostate cancer metastasized patient; PCa-l, prostate cancer localized.

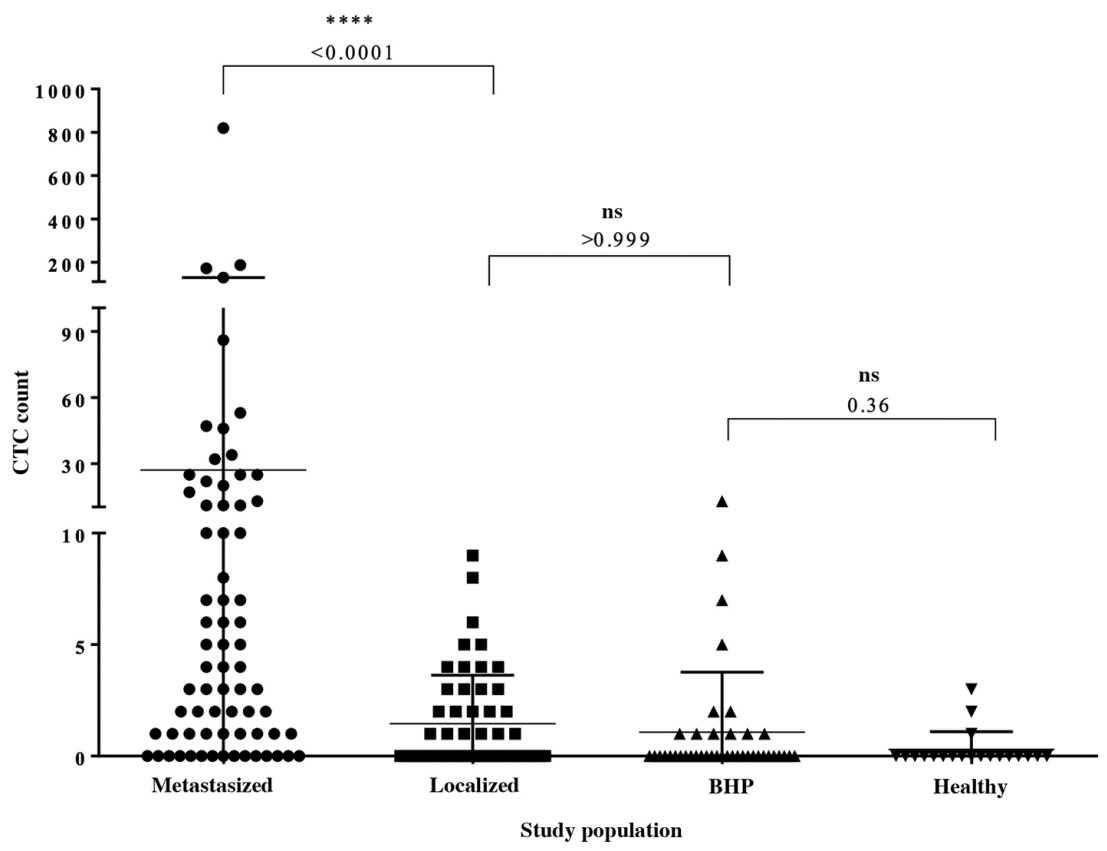

Figure 3. CTC enumeration in metastasized and localized PCa patients and the control groups as evaluated with the CellCollector ${ }^{\circledR}$. Data are shown as the median and interquartile range. Dunn's multiple comparisons test was used to calculate the P-value. PCa, prostate cancer.

also detected in the female controls. With the exception of the female control group, one wire per group from the PCa-m, PCa-1 and $\mathrm{BPH}$ groups could not be evaluated.

Effectivity of CTC isolation technologies. For direct comparison of the CTC isolation technology, 95 analyzable blood samples ( $7.5 \mathrm{ml}$ CellSave) were taken prior to the CellCollector applications and detected by the CellSearch system. As shown in Fig. 4, the CellCollector captured in vivo CTCs in 18 of 39 PCa-l patients $(46.2 \%)$ with a median (range) of $0(0-9)$; in PCa-m patients, the CellCollector detected CTCs in 47 of 60 patients $(78.4 \%)$ with a median (range) of $3(0-820)$ CTCs.

The CellSearch system isolated CTCs in 4 of 39 of the PCa-1 patients $(10.3 \%)$ with a median (range) of $0(0-1)$ and showed positive results for CTCs in 40 of 60 of the $\mathrm{PCa}-\mathrm{m}$ patients $(67 \%)$ with a median (range) of $3.5(0-1,428)$ (Fig. 4). Both systems demonstrated no correlation between CTC counts.

To investigate the diagnostic accuracy of the CellCollector, we compared the PSA level and CTCs detected using the CellSearch system in $\mathrm{PCa}-\mathrm{m}$ patients, $\mathrm{BPH}$ patients and healthy donors. Notably, the ROC curves for these three parameters showed similar areas under the curve (AUCs): 0.87 (95\% CI, 0.8-0.94) for PSA, 0.82 (95\% CI, 0.74-0.89) for the CellCollector and 0.84 (95\% CI, 0.76-0.92) for the CellSearch system (Fig. 5). These values indicated a low likelihood of false-negative and false-positive results.

Individual patient CTC/PSA profiles. Regarding the individual CTC/PSA profiles for therapy monitoring in the PCa-m group, we included only metastasized patients with more than three visits. The CTC/PSA profiles over time are illustrated for 4 patients in Fig. 6A-D. The onset of hormonal therapy in patient P072 led to a short decline in the PSA level and CTC count. Approximately one month before visit 8, chemotherapy with docetaxel was started. This patient presented radiologic and PSA progression and a rising CTC count (Fig. 6B). As observed in patient P022, the CTC number and the PSA level were clearly associated with additional therapies, such as palliative transurethral resection of the prostate (TURP) and removal of brain metastases (Fig. 6D). However, the PSA and CTC profiles could be related to the need for additional therapy in only 4 of the 10 patients with metastatic disease (Fig. 6A-D).

Correlation of in vivo CTC count with clinical outcome and CTC kinetics. The median follow-up time in the cancer group was 37 months. The Kaplan-Meier curves, according to the CTC count for metastatic prostate patients, showed that a CTC count of 5 or more has a lower median OS of 


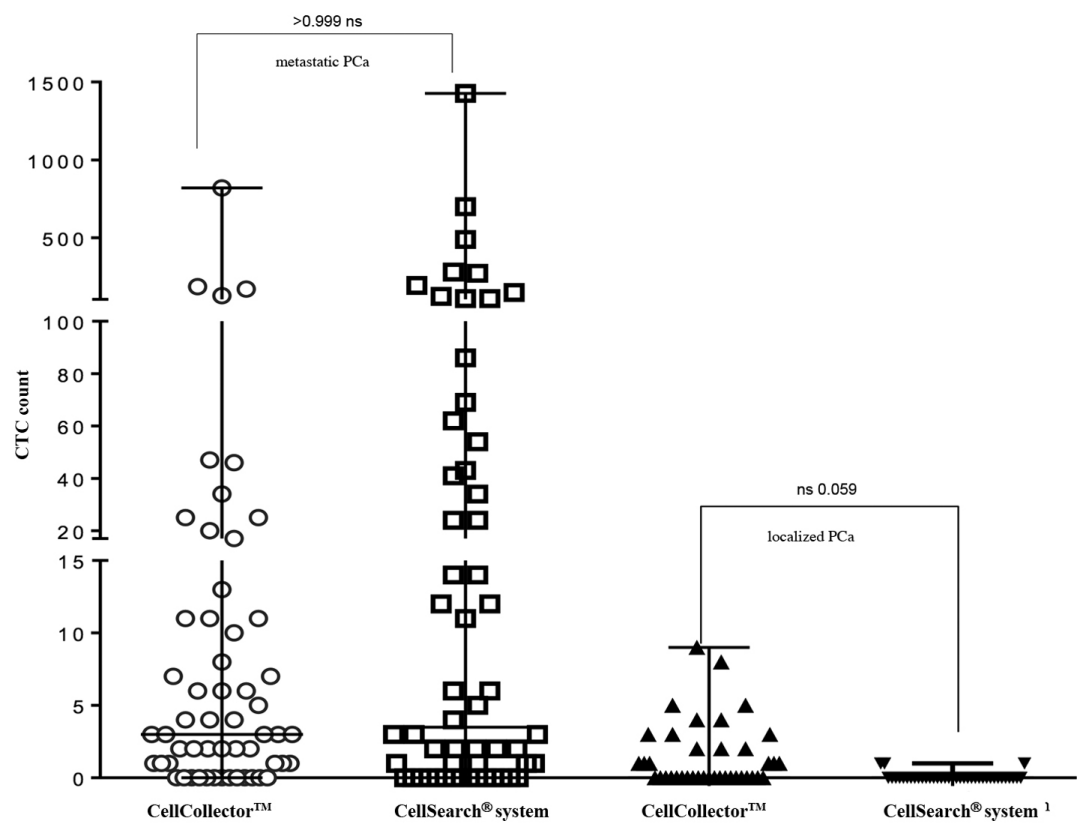

Figure 4. Comparison of CTC isolation technologies (CellCollector ${ }^{\circledR}$ and CellSearch ${ }^{\circledR}$ system). The results of CTC enumeration are summarized for PCa patients after a 12-month follow-up period. A median of 3 CTCs in PCa-m and a median of 0 CTCs in PCa-1 were detected with the CellCollector. The CellSearch system detected a median of 3.5 CTCs in PCa-m and a median of 0 in PCa-1, respectively. Data are shown as the median and interquartile range. PCa, prostate cancer; CTCs, circulating tumor cells; PCa-1, prostate cancer localized; PCa-m, prostate cancer metastasized.

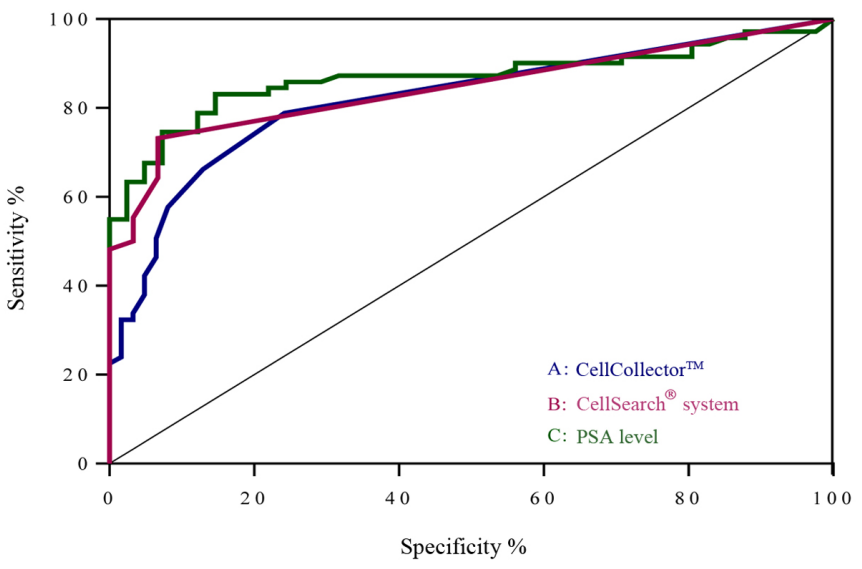

\begin{tabular}{lccc} 
& AUC & $\mathbf{9 5 \%}$ CI & P*** \\
\hline A: PSA level $(\mathbf{n g} / \mathbf{m l})$ & 0.87 & $0.80-0.94$ & $<0.0001$ \\
B: CellCollector & & & \\
& 0.85 & $0.76-0.92$ & $<0.0001$ \\
C: CellSearch $^{\circledast}$ system & 0.82 & $0.74-0.89$ & $<0.0001$
\end{tabular}

Figure 5. ROC curves illustrating diagnostic accuracy of CTCs count detected with the CellCollector ${ }^{\circledR}$ (blue line), with CellSearch ${ }^{\circledR}$ system (pink line) and the PSA level (green line) of metastasized prostate cancer patients to control group. Results of ROC analysis curves for (A) CellCollector, (B) CellSearch system, and (C) PSA level (ng/ml). ROC, receiver operating characteristic; CTCs, circulating tumor cells; PSA, prostate specific antigen.

27.5 months compared to 37 months for patients with less than 5 CTCs (HR 2.6, 95\% CI, 0.78-8.3) (Fig. 7A). Interestingly, PCa-m patients with more than 5 CTCs at all time points and increasing CTC counts showed the shortest median OS of 25 months compared to 34 months (HR 1.9, 95\% CI 0.4-11.6) for patients with declining CTC counts (Fig. 7B).
In a match analysis of localized $\mathrm{PCa}$ patients before and after RP, 6 of 14 patients (43\%) showed at least one CTC. The 6- and 12 -month visits after surgery included $8(58 \%)$ and $5(36 \%)$ patients who were CTC-positive. In the follow-up of PCa-1 patients after RP, we observed no significant variation in the CTC number and no prognosis of OS. Furthermore, the presence of CTCs did not correlate with the PSA level, Gleason score or tumor, node, metastasis (TNM) classification in any PCa group.

\section{Discussion}

The development of sensitive and specific assays for the detection, isolation and characterization of CTCs in the blood of cancer patients is still in progress. At this time, only one assay (CellSearch system) has achieved the level of validity for approval by the FDA (11).

Our objective was to prove the clinical feasibility of the in vivo isolation of CTCs in PCa patients. The CellCollector was inserted into the vein of the patient six times per year per patients with metastatic prostate cancer. The wire was well tolerated in patients and the control group.

We demonstrated a significant difference between in vivo-captured CTCs in localized (median $=0$ CTCs) and metastasized PCa (median, 4 CTCs) patients (Fig. 3). According to our data, CTC counts relative to the criterion threshold of five-displayed individual association with overall survival in metastasized PCa patients (Fig. 7A). This threshold status of more than five CTCs was validated in MPCa patients by the CellSearch system (11). Metastasized prostate cancer patients who showed rising CTC counts at all-time points demonstrated an OS period of 25 months compared to 34 months for those who showed declining CTCs (Fig. 7B). Our data demonstrated that a rinsing CTC count may be involved in a cancer progression resulting in active tumor spreading. 
A

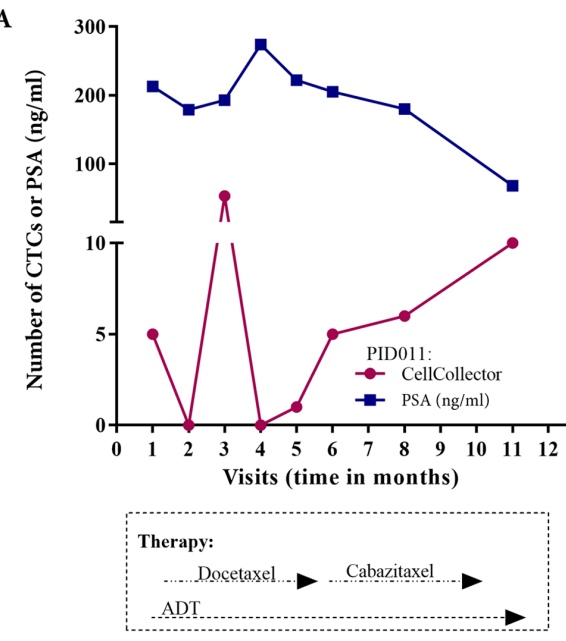

C

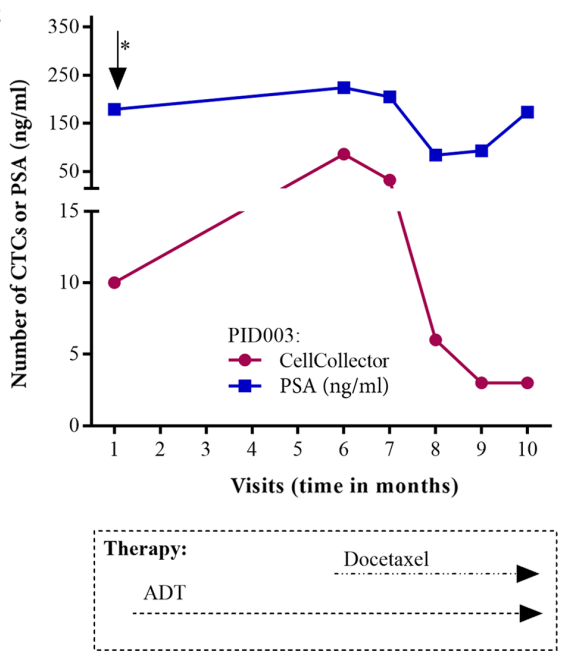

B
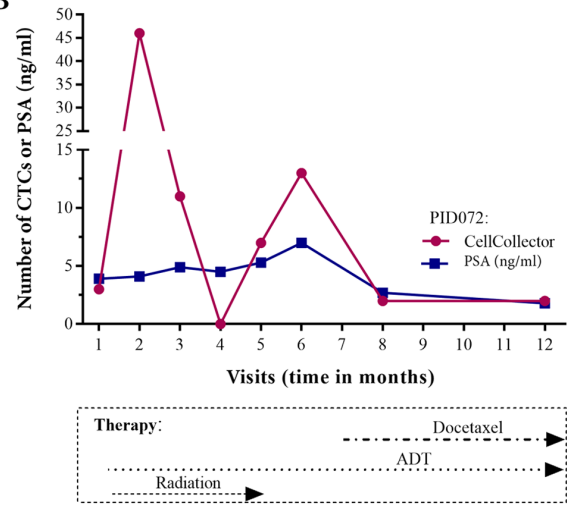

D
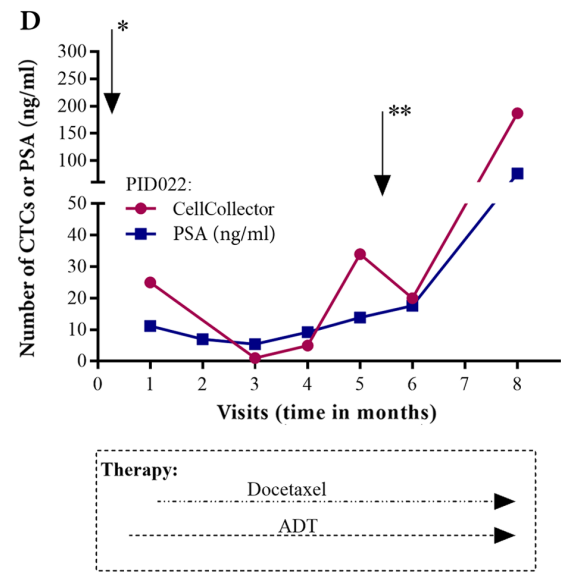

Figure 6. Treatment history and CTC/PSA profiles of 4 metastasized PCa patients, which fulfill the inclusion criteria, up to 12 months. (A) Patient enrolled in the study 4 months after palliative TURP (B) Patient with highly differentiated adenocarcinoma 18 years after prostatectomy. (C) Patient included one month after palliative TURP $\left(^{*}\right)$ and showing continuous hormone therapy and disease progression. (D) Only 6 days after study inclusion, palliative TURP ${ }^{*}$ ) was performed with continuous hormone therapy and radiotherapy and removal of a brain metastasis between months 5 and $6\left({ }^{* *}\right)$. CTCs, circulating tumor cells; PSA, prostate specific antigen; PCa, prostate cancer; TURP, transurethral resection of the prostate.

A

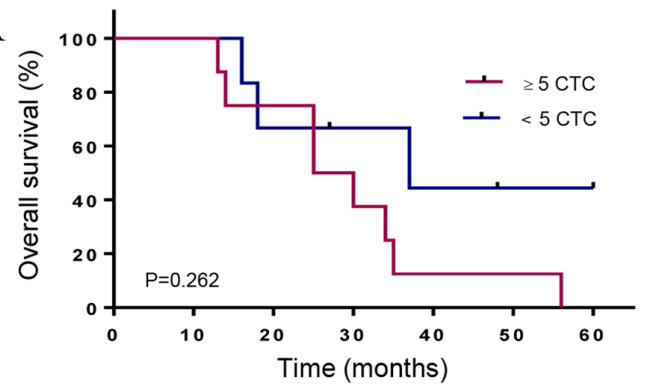

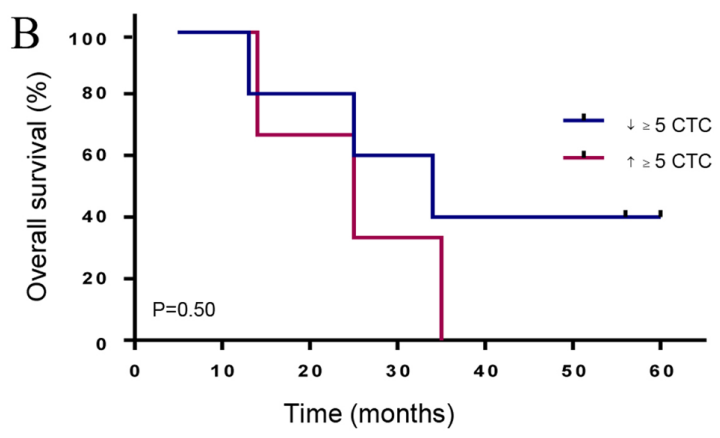

Figure 7. Kaplan-Meier overall survival according to CTCs. (A) Patients with metastatic prostate cancer (PCa-m) with $\geq 5 \mathrm{CTCs}$ and $<5$ CTCs showed differences in OS (27.5 months vs. 37 months [HR 2.6, 95\% CI, 0.78-8.3]). (B) CTC kinetics for PCa-m patients with $\geq 5$ CTCs at all-time points; the median OS differed between those with declining CTC ( $\downarrow \geq 5$ CTCs) counts and those with increasing CTC ( $\uparrow \geq 5$ CTCs) counts ( 25 months vs. 34 months, HR 1.9, 95\% CI, 0.4-11.6), HR, hazard ratio; OS, overall survival; CTCs, circulating tumor cells.

In the current investigation, we further revealed that the area under the curve (AUC) value did not differ significantly between the in vivo captured CTC and PSA levels and ex vivo captured CTCs (Fig. 5). This indicates a similar diagnostic performance of the CTC count and PSA level. In contrast,
Goldkorn et al (21) demonstrated in the SWOGS0421 trial that the ROC curves for the day- 0 CTC count had considerably higher AUCs than those for the day-0 PSA level. One possible reason could be that CTCs, in contrast to PSA, are not directly affected by hormonal treatment. 
Furthermore, we were able to measure the CTC/PSA profile in metastatic prostate cancer patients during therapeutic layering. Importantly, the individual profiles showed promise for therapeutic decision-making in this patient group (Fig. 6). This offers the opportunity for disease monitoring and for making a tailored treatment decision in individual metastatic prostate cancer patients. In the area of personalized medicine, such profiling allows to protect patients from unnecessary side effects of ineffective treatment. Our results confirm the potential of CTCs as pharmacodynamics and potential intermediate endpoint biomarkers for overall survival $(7,11)$. Unfortunately, this option is not possible in all our PCa-m patients, partially due to the heterogeneous phenotypes of CTCs in PCa patients. These phenotypes reflect the epithelial-mesenchymal transition (EMT) process, which plays a critical role in cancer metastasis. Indeed, CTCs undergo phenotypic changes from epithelial to more mesenchymal transitional states during the metastatic transition (22-24). An enhancement of the in vivo CTC isolation in metastasized prostate cancer patients should be considered to use an EMT stable marker such as Prostate-Specific Membrane Antigen (PSMA) (25) for functionalizing the CellCollector.

Interestingly, in the metastasized group, the detection rate of $79.2 \%$ was slightly reduced compared to the $100 \%$ rate of ex vivo CTC isolation in our previous study (14). An explanation (as described above) could be the heterogeneous phenotypes of CTCs in patients with progressive cancer status. Furthermore, we detected more leucocytes and cells positive for EpCAM, CD45 and pan-CK (data not shown) in comparison to the ex vivo application of the wire (14). It remains challenging to examine and analyze CTCs, as this phenomenon indicates activation of the immune system and may signal a therapeutic response (9). The results of our trial are in general agreement with previous results of other CTC trials in PCa-m patients (11,26-28).

The role of CTCs in PCa-1 has been proposed as an option for postoperative risk stratification. We isolated CTCs in $45.3 \%$ of the wire applications in $\mathrm{PCa}-1$ patients. The monitoring of the CTC count ( $>1$ CTC) before and after radical prostatectomy demonstrated no correlation with clinical and pathological parameters. Meyer et al (29) analyzed 152 localized patients preoperatively for CTCs and reported an 11\% $(n=17)$ CTC-positive rate. They showed no difference in biochemical recurrence in patients with or without CTCs. Todenhofer et al (30) used an EpCAM-independent isolation system and detected CTCs in $50 \%$ of $\mathrm{PCa}-1$ patients. They also revealed no correlation between CTC detection and PSA level, tumor stage or Gleason score (30). Taken together, our results did not verify a significant association of CTC positivity in patients undergoing RP for preoperative localized $\mathrm{PCa}$. Our control groups also demonstrated a median CTC of 0 , which was similar to the median CTC of the local PCa group. We demonstrated in Fig. 5 a good diagnostic accuracy of the CellCollector for CTC capturing comparable to the CellSearch System and the PSA level. The in vivo CTC isolation system allows to detect the low number of cells. From the other hand, it create the risk of the false-positive results. The localized prostate cancer group consist of $85.7 \%$ patients with a low Gleason score $\leq 7$. It is very unlikely that such tumors release high invasive CTCs. False-positive results in control groups (BPH and healthy women) can have different causes. One reason may be the used characterization of CTCs, which does not distinguish between possible different CTCs phenotypes. CTCs can infiltrate normal tissue and form metastases or, which is more likely, be attacked by immune cells and killed. It is also possible to misinterpret cells, like larger CD45 negative leucocytes or low number of epithelial cells, which potentially enter in blood by the peripheral intravenous cannulation. Castle et al (31) discussed also such subtype of cells, which remained to be characterized. In our previous in vitro study, we demonstrated that $54 \%$ of the BPH patients' blood samples are positive for CTCs (14). In the present trail, only $20.3 \%$ of the $\mathrm{BPH}$ patients were positive for CTCs. This might suggest a better diagnostic accuracy of the in vivo CTC isolation compared the ex vivo CellCollector application (14). Our findings suggest that a cutoff value of in vivo captured CTCs must be developed. The role of CTCs as prognostic markers in localized PCa seems to be more relevant in locally advanced prostate cancer patients, such as risk stratification for additional treatment.

The limitations of our investigation are of course the small number of patients in the cancer groups. The unknown blood volume of the reported CTC counts must also be noted. Thus, future studies are needed to discover the clinical potential of the wire.

In conclusion, our present findings indicate that in vivo capture of CTCs by the CellCollector overcomes the blood volume limitations of other diagnostic approaches and thereby increases the diagnostic sensitivity of CTC analysis. The CTC/PSA-Profile reveals the possibility of personalized therapy monitoring which can help to prevent patients for side effects of invalidly treatment. The CellCollector was well tolerated, and no side effects were reported. Thus, future studies are needed to explore this method in the clinic.

\section{Acknowledgements}

The authors would like to thank the Department of Tumor Biology, University Medical Center Hamburg-Eppendorf, Hamburg for providing the CellSearch analysis.

\section{Funding}

Financial funding for the implementation of this study was received from the GILUPI GmbH. The sponsor played no role in the study.

\section{Availability of data and materials}

The datasets used and/or analyzed during the current study are available from the corresponding author on reasonable request.

\section{Authors' contributions}

GT designed the study, performed the experiments, analyzed the data and wrote the manuscript. GT and PF confirm the authenticity of all the raw data. $\mathrm{CB}$ and KF performed the experiments and were involved in drafting the paper. JB performed the analysis and interpretation of data, and was involved in drafting the paper and revised it critically for important intellectual content. RH and EW designed the study. FK and SS performed the analysis and interpretation of data, and gave final approval of the version to be published. The conception and design of the study, as well as drafting and approving the article, is attributed to PF. All authors read and approved the final manuscript. 


\section{Ethics approval and consent to participate}

The study protocol was approved by the Medical Faculty Ethics Committee of MLU Halle-Wittenberg. Furthermore, we obtained a permit from the Federal Institute for Drug and Medical Devices (Germany, BfArM). The patients provided written informed consent.

\section{Patient consent for publication}

Not applicable.

\section{Competing interests}

The authors declare that GILUPI GmbH supported the present study. We received funding from GILUPI GmbH; they provided the CellCollector and the patients received travel expenses. The sponsor played no role in the study. GILUPI $\mathrm{GmbH}$ provided the schematic image of the wire (Fig. 1).

\section{Authors' information}

Raschid Hoda was employed until February 2013 at the Medical Faculty of Martin Luther University Halle-Wittenberg, University Clinic and Outpatient Clinic for Urology, 06120 Halle (Saale), Germany.

\section{References}

1. Bray F, Ferlay J, Soerjomataram I, Siegel RL, Torre LA and Jemal A: Global cancer statistics 2018: GLOBOCAN estimates of incidence and mortality worldwide for 36 cancers in 185 countries. CA Cancer J Clin 68: 394-424, 2018.

2. Nuhn P, De Bono JS, Fizazi K, Freedland SJ, Grilli M, Kantoff PW, Sonpavde G, Sternberg CN, Yegnasubramanian S and Antonarakis ES: Update on systemic prostate cancer therapies: Management of metastatic castration-resistant prostate cancer in the era of precision oncology. Eur Urol 75: 88-99, 2019.

3. Resnick MJ, Koyama T, Fan KH, Albertsen PC, Goodman M, Hamilton AS, Hoffman RM, Potosky AL, Stanford JL, Stroup AM, et al: Long-term functional outcomes after treatment for localized prostate cancer. N Engl J Med 368: 436-445, 2013.

4. Scher HI, Lu D, Schreiber NA, Louw J, Graf RP, Vargas HA, Johnson A, Jendrisak A, Bambury R, Danila D, et al: Association of AR-V7 on circulating tumor cells as a treatment-specific biomarker with outcomes and survival in castration-resistant prostate cancer. JAMA Oncol 2: 1441-1449, 2016.

5. Hofman P and Popper HH: Pathologists and liquid biopsies: To be or not to be? Virchows Arch 469: 601-609, 2016.

6. Alix-Panabieres $\mathrm{C}$ and Pantel $\mathrm{K}$ : Challenges in circulating tumour cell research. Nat Rev Cancer 14: 623-631, 2014.

7. Yap TA, Lorente D, Omlin A, Olmos D and de Bono JS: Circulating tumor cells: A multifunctional biomarker. Clin Cancer Res 20: 2553-2568, 2014.

8. Antonarakis ES, Lu C, Wang H, Luber B, Nakazawa M, Roeser JC, Chen Y, Mohammad TA, Chen Y, Fedor HL, et al: AR-V7 and resistance to enzalutamide and abiraterone in prostate cancer. N Engl J Med 371: 1028-1038, 2014.

9. Andree KC, van Dalum G and Terstappen LW: Challenges in circulating tumor cell detection by the CellSearch system. Mol Oncol 10: 395-407, 2016.

10. Cristofanilli M, Budd GT, Ellis MJ, Stopeck A, Matera J, Miller MC, Reuben JM, Doyle GV, Allard WJ, Terstappen LW and Hayes DF: Circulating tumor cells, disease progression, and survival in metastatic breast cancer. N Engl J Med 351: 781-791, 2004.

11. de Bono JS, Scher HI, Montgomery RB, Parker C, Miller MC, Tissing H, Doyle GV, Terstappen LW, Pienta KJ and Raghavan D: Circulating tumor cells predict survival benefit from treatment in metastatic castration-resistant prostate cancer. Clin Cancer Res 14: 6302-6309, 2008.
12. Habli Z, AlChamaa W, Saab R, Kadara H and Khraiche ML: Circulating tumor cell detection technologies and clinical utility: Challenges and opportunities. Cancers (Basel) 12: 1930, 2020.

13. Ferreira MM, Ramani VC and Jeffrey SS: Circulating tumor cell technologies. Mol Oncol 10: 374-394, 2016.

14. Theil G, Fischer K, Weber E, Medek R, Hoda R, Lucke K and Fornara P: The use of a new CellCollector to isolate circulating tumor cells from the blood of patients with different stages of prostate cancer and clinical outcomes-a proof-of-concept study. PLoS One 11: e0158354, 2016.

15. Gorges TM, Penkalla N, Schalk T, Joosse SA, Riethdorf S, Tucholski J, Lücke K, Wikman H, Jackson S, Brychta N, et al: Enumeration and molecular characterization of tumor cells in lung cancer patients using a novel in vivo device for capturing circulating tumor cells. Clin Cancer Res 22: 2197-2206, 2016.

16. Saucedo-Zeni N, Mewes S, Niestroj R, Gasiorowski L, Murawa D, Nowaczyk P, Tomasi T, WeberE,DworackiG,Morgenthaler NG, et al: A novel method for the in vivo isolation of circulating tumor cells from peripheral blood of cancer patients using a functionalized and structured medical wire. Int J Oncol 41: 1241-1250, 2012.

17. Mandair D, Vesely C, Ensell L, Lowe H, Spanswick V, Hartley JA, Caplin ME and Meyer T: A comparison of cellcollector with cellsearch in patients with neuroendocrine tumours. Endocr Relat Cancer 23: L29-L32, 2016.

18. de Bono JS, Attard G, Adjei A, Pollak MN, Fong PC, Haluska P, Roberts L, Melvin C, Repollet M, Chianese D, et al: Potential applications for circulating tumor cells expressing the insulin-like growth factor-I receptor. Clin Cancer Res 13: 3611-3616, 2007.

19. Cristofanilli M: Circulating tumor cells, disease progression, and survival in metastatic breast cancer. Semin Oncol 33 (Suppl 9): S9-S14, 2006.

20. Allard WJ, Matera J, Miller MC, Repollet M, Connelly MC, Rao C, Tibbe AG, Uhr JW and Terstappen LW: Tumor cells circulate in the peripheral blood of all major carcinomas but not in healthy subjects or patients with nonmalignant diseases. Clin Cancer Res 10: 6897-6904, 2004.

21. Goldkorn A, Ely B, Quinn DI, Tangen CM, Fink LM, Xu T, Twardowski P, Van Veldhuizen PJ, Agarwal N, Carducci MA, et al: Circulating tumor cell counts are prognostic of overall survival in SWOG S0421: A phase III trial of docetaxel with or without atrasentan for metastatic castration-resistant prostate cancer. J Clin Oncol 32: 1136-1142, 2014.

22. Armstrong AJ: Epithelial-mesenchymal transition in cancer progression. Clin Adv Hematol Oncol 9: 941-943, 2011.

23. Alix-Panabieres $C$ and Pantel K: Circulating tumor cells: Liquid biopsy of cancer. Clin Chem 59: 110-118, 2013.

24. Lambert AW, Pattabiraman DR and Weinberg RA: Emerging biological principles of metastasis. Cell 168: 670-691, 2017.

25. Gleghorn JP, Pratt ED, Denning D, Liu H, Bander NH, Tagawa ST, Nanus DM, Giannakakou PA and Kirby BJ: Capture of circulating tumor cells from whole blood of prostate cancer patients using geometrically enhanced differential immunocapture (GEDI) and a prostate-specific antibody. Lab Chip 10: 27-29, 2010.

26. Danila DC, Anand A, Sung CC, Heller G, Leversha MA, Cao L, Lilja H, Molina A, Sawyers CL, Fleisher M and Scher HI: TMPRSS2-ERG status in circulating tumor cells as a predictive biomarker of sensitivity in castration-resistant prostate cancer patients treated with abiraterone acetate. Eur Urol 60: 897-904, 2011.

27. Lorente D, Olmos D, Mateo J, Bianchini D, Seed G, Fleisher M, Danila DC, Flohr P, Crespo M, Figueiredo I, et al: Decline in circulating tumor cell count and treatment outcome in advanced prostate cancer. Eur Urol 70: 985-992, 2016.

28. Scher HI, Heller G, Molina A, Attard G, Danila DC, Jia X, Peng W, Sandhu SK, Olmos D, Riisnaes R, et al: Circulating tumor cell biomarker panel as an individual-level surrogate for survival in metastatic castration-resistant prostate cancer. J Clin Oncol 33: 1348-1355, 2015.

29. Meyer CP, Pantel K, Tennstedt P, Stroelin P, Schlomm T, Heinzer H, Riethdorf S and Steuber T: Limited prognostic value of preoperative circulating tumor cells for early biochemical recurrence in patients with localized prostate cancer. Urol Oncol 34: 235.e11-6, 2016.

30. Todenhofer T, Park ES, Duffy S, Deng X, Jin C, Abdi H, Ma H and Black PC: Microfluidic enrichment of circulating tumor cells in patients with clinically localized prostate cancer. Urol Oncol 34: 483.e9-e16, 2016.

31. Castle J, Morris K, Pritchard S and Kirwan CC: Challenges in enumeration of CTCs in breast cancer using techniques independent of cytokeratin expression. PLoS One 12: e0175647, 2017.

This work is licensed under a Creative Commons Attribution-NonCommercial-NoDerivatives 4.0 International (CC BY-NC-ND 4.0) License. 